Article

\title{
Framework for Managing Maintenance of Wind Farms Based on a Clustering Approach and Dynamic Opportunistic Maintenance
}

\author{
Juan Izquierdo ${ }^{1,2, *(\mathbb{C})}$, Adolfo Crespo Márquez ${ }^{2}$, Jone Uribetxebarria ${ }^{1}$ and Asier Erguido ${ }^{1,2}$ (1) \\ 1 Ikerlan Technology Research Centre, Operations and Maintenance Technologies Area, \\ 20500 Gipuzkoa, Spain; juribetxebarria@ikerlan.es (J.U.); aerguido@ikerlan.es (A.E.) \\ 2 Industrial Organization and Business Management I, School of Engineering, University of Seville, \\ Camino de los Descubrimientos s/n, 41092 Seville, Spain; adolfo@us.es \\ * Correspondence: jizquierdo@ikerlan.es
}

Received: 15 April 2019; Accepted: 20 May 2019; Published: 28 May 2019

\begin{abstract}
The growth in the wind energy sector is demanding projects in which profitability must be ensured. To fulfil such aim, the levelized cost of energy should be reduced, and this can be done by enhancing the Operational Expenditure through excellence in Operations \& Maintenance. There is a considerable amount of work in the literature that deals with several aspects regarding the maintenance of wind farms. Among the related works, several focus on describing the reliability of wind turbines and many set the spotlight on defining the optimal maintenance strategy. It is in this context where the presented work intends to contribute. In the paper a technical framework is proposed that considers the data and information requisites, integrated in a novel approach a clustering-based reliability model with a dynamic opportunistic maintenance policy. The technical framework is validated through a case study in which simulation mechanisms allow the implementation of a multi-objective optimization of the maintenance strategy for the lifecycle of a wind farm. The proposed approach is presented under a comprehensive perspective which enables the discovery an optimal trade-off among competing objectives in the Operations \& Maintenance of wind energy projects.
\end{abstract}

Keywords: maintenance management; wind turbines; clustering; reliability; dynamic opportunistic maintenance; simulation

\section{Background and Introduction}

The attention drawn by renewable energy has increased considerably over recent years. This increasing importance has nurtured an important growth, which is especially prominent in the wind energy sector [1]. Wind energy is one of the main sources of power generation in Europe with a vast majority of installed capacity in the form of onshore Wind Farms (WFs) [2]. For the profitability of wind energy projects to be ensured, it is essential to reduce the levelized cost of energy (LCoE) to its minimum [2,3]. Aiming at increasing the energy yield of WFs, the LCoE should be reduced, and this can be done by directly addressing the Capital Expenditure (CAPEX) and the Operational Expenditure (OPEX) of the projects [2].

The scope of the research here presented abides in OPEX reduction; more specifically, this paper is intended to reduce the Operations and Maintenance (O\&M) costs. How to reduce the O\&M costs has become an ongoing challenge for WFs; the figures associated with these costs are notorious $[4,5]$ and may rise by up to $32 \%$ and $12-30 \%$ for offshore and onshore WFs, respectively $[6,7]$. However, aiming at lowering the LCoE by reducing O\&M costs is a two-fold challenge since it also entails minimizing the lost energy production [8] for the entire lifecycle, which oscillates around 20 years perspective [9]. According to the International Renewable Agency, the costs associated with O\&M 
account for up to one quarter of the proportion of the LCoE, with $80 \%$ of this expenses directly attributed to maintenance [10-12].

In view of the maintenance role in the LCoE and thus in the profitability of WFs, it is important to consider models for optimization of O\&M plans and decisions [2]. The evolution of maintenance models and methodologies have kept pace with the constant technological evolution of Wind Turbines (WTs) [3]. According to [3], the goal of all the approaches and methodologies is determining the most adequate maintenance plan, the management of the resources, and the aspects related to Reliability, Availability, and Maintainability (RAM) of the WTs.

Within such context, the WF operators are bound to develop new techniques and decision-support tools for optimal maintenance strategies, if they strive to maximize the profitability of the investment [8]. Accordingly, maintenance management discipline acquires a highly significant position since it provides a comprehensive perspective for the management of WTs, allowing for optimal maintenance strategies which reduce maintenance costs while maximizing availability $[3,5]$.

The decision-making process in the asset management field has been divided into strategic (long-term), tactic (medium-term), and operational (short-term) to achieve excellence in maintenance [13-15]. It is the purpose of the research in this paper to address the different aspects of the maintenance decision-making process. This is done by answering the research question of whether it is possible to achieve maintenance excellence for the lifecycle of WFs by a maintenance strategy which considers the different behaviors of the WTs and integrated business-related objectives.

Aiming at providing an answer for the aforementioned research question, a technical framework for managing maintenance is proposed in the paper. The framework is a comprehensive proposal that considers different aspects regarding the maintenance of a WF. Within the possibilities offered by the current trend for big data, certain key aspects to create a failure database are integrated in the framework, which enables a clustering approach based on the failure behaviors of the different failure modes of each WT. This approach supports an opportunistic maintenance policy with dynamic thresholds that regard not only to the reliability of the assets but also business considerations. Besides, the framework also incorporates the strategic view through a Life-Cycle Cost (LCC) perspective integrated by means of a multi-objective optimization and supported by simulation techniques that provide valuable information to find an attractive trade-off between cost and performance.

\subsection{Related Works}

In the context of maintenance, several studies have proven the utility of reliability approaches to optimize it $[5,16,17]$. If seeking to take forward a reliability study, the information needed as an input to the decision should be at hand, in the right format, and on time [18]. The idea of a common database in the wind energy sector is not novel. In [19] the objectives of a RAM database can be seen and in [10] different sources of WT data are analyzed. The challenges faced when building such database with quality data have been addressed by the integration of data coming from different sources $[4,10,19,20]$. Nonetheless, to translate the data into information and exploit its inherent value, it is essential a correct assessment of the failure process and therefore the selection of the proper time-to-failure model, which will enable optimization of the maintenance plan [21].

The reliability study of a WF and the creation of the failure database involves combining data from similar assets, in this case WTs. This combination of data is known as data-pooling, and in [21], as well as in [22], several conditions to be met by the equipment subject to data-pooling are stated. Nonetheless, this may lead to combining data from assets with unlike behaviors. The heterogeneity in the failure behavior among WTs may be caused by the presence of different models of WTs, which entail different technical solutions [23]. Moreover, this multiplicity in the failure frequencies may also be the result of the different operational conditions of the assets $[16,17,24,25]$.

To deal with the diversity in failure behaviors when managing a considerable number of assets, similarity-based approaches have been proposed in recent research works [26-28]. The work in [27] where a spectral clustering approach is proposed to later address the maintenance optimization 
problem to all the assets belonging to the same cluster is especially interesting. Another interesting work is the research in [29], which provides a very similar approach using a different clustering algorithm. In the wind energy sector, and with the purpose of enhancing maintenance management, approaches based on clustering concepts and algorithms can also be found in the recent literature. A fuzzy clustering approach based on Mahalanobis distance is proposed in [30] for warnings and failure detection and it is applied to a real WF. Another interesting proposal is the cluster analysis combined with Frequent Pattern Mining presented in [31] for WT fault detection. In addition, it has also been proposed along with Artificial Neural Networks in [32] for developing optimal maintenance strategies.

The selected approach highly conditions the adequacy of the maintenance strategy [33]. In the case of WTs, it is important to select one that takes into account the multi-component nature of the turbines [34,35]. The WTs are composed of several subsystems with dependencies among them that can be classified as (i) economic, where the simultaneous performance of maintenance activities implies different economic consequences that implement them individually [36]; (ii) structural, where maintenance actions on one system may imply maintenance activities on others [37]; and (iii) stochastic, where the failure hazard of two different systems are not independent [38]. In this context, opportunistic maintenance policies are the most suitable, and therefore they have been widely researched [33].

Opportunistic maintenance policy makes the most of short-term situations to perform the maintenance of non-failed systems when a failure has already happened in another one, grounding its decision on a threshold regarding a system's age, reliability or health condition [5]. Opportunistic maintenance has proven its utility in the sector through several works in the recent literature [39], and it has been highly related to multi-criteria approaches [40]. The consideration of opportunistic maintenance under a multi-criteria perspective allows the handling of some main conflicting objectives such as maintenance cost, availability, or manager preferences [41]. This advantage is especially beneficial in the wind energy sector where it is important to bear in mind the maximization of revenue, power, and reliability, and the minimization of Operations and Maintenance costs [42]. Some of the latest research in the wind energy sector is in this area. The application of opportunistic maintenance in [43] takes advantage of low wind-speed periods to perform corrective actions. The research in [44] provides an opportunistic maintenance policy based on remaining useful life estimation according to condition-monitoring data. The opportunistic maintenance is also proposed with different types of maintenance actions, e.g., the works in [34,45-47]. In addition, the authors of [48,49] integrate the opportunistic maintenance policy with multi-objective optimization.

\subsection{Overview}

Given the importance of the reviewed works in the literature of the Wind Energy sector, it is the scope of this paper to provide a managing framework that supports the maintenance management of WF operators. The proposed framework is a cross-functional value proposition that starts by considering the requisites of the failure database to later define a maintenance policy. The opportunistic maintenance policy will be supported by a clustering approach that allows the addressing of different behaviors of the WTs within the same WF. Besides, the framework is considered under a lifecycle perspective integrated through simulation techniques and multi-objective optimization algorithms.

The proposed framework is presented in the following Section 2. An initial and brief introduction is first provided along with a representation according to IDEF0 methodology, then every function is explained in detail in each corresponding subsection. The principles of the creation of a failure data base for the study is explained in Section 2.1, the reliability considerations of WTs are presented in Section 2.2, and finally, the opportunistic maintenance policy is explained in Section 2.3. In Section 3 the case study is presented, Section 3.1 describes the development of the case study and the initial assumptions and then Section 3.2 consists of the results obtained in the implementation of the framework. Finally, Section 4 summarizes the main conclusions obtained through the research 
process, its output represented as the framework, and the application to a real case study in the wind energy sector.

\section{Framework and Methods}

The proposed technical framework, Figure 1, is a comprehensive integration of different technical solutions and methods to ease maintenance management. The framework proposes an initial data treatment, considering different information sources, to create a RAM database that will enable modeling of the reliability of WTs. To undertake the reliability modeling, a clustering algorithm is proposed along with the Kullback-Leibler Divergence measure, which approach addresses the difficulty derived from the heterogeneity in the failure behaviors of the WTs. The reliability models, which describe the failure behaviors within the WF, as well as data from the RAM database and information regarding the cost structure, will serve as input for the methods proposed for defining an optimal maintenance strategy. These methods regard multi-objective optimization algorithms and simulation software under both a lifecycle perspective and governed by the principles of opportunistic maintenance.

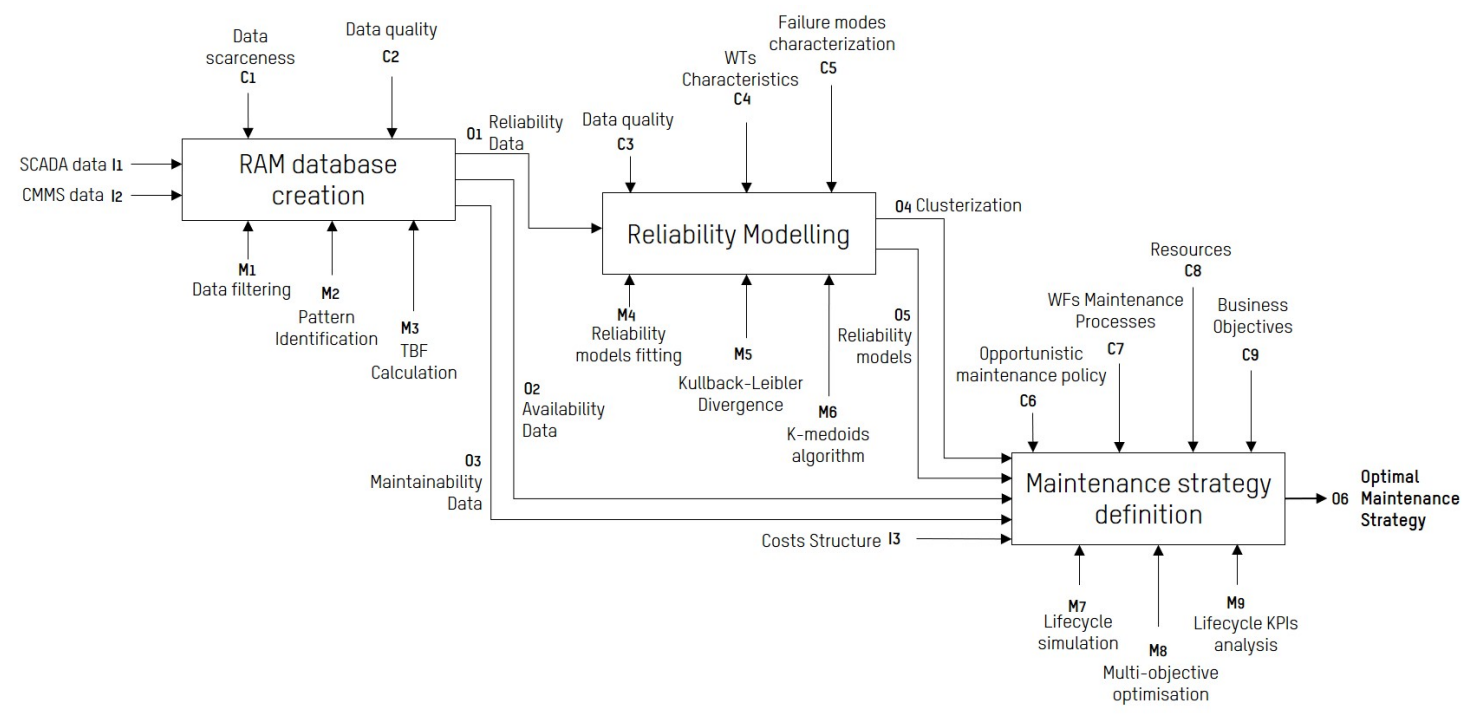

Figure 1. Technical framework.

The technical framework is represented in Figure 1 following the IDEF0 methodology [50], it consists of three functions: RAM database creation, Reliability modeling, and Maintenance strategy definition. The functions are associated with interfaces represented by arrows, which have been referred to as INCOMs [51]: Inputs (I) of the function box enter from the left, they are transformed into Outputs $(\mathrm{O})$ leaving the box by means of the Mechanisms (M), which enter though the bottom and refer to tools, algorithms, or resources that enable the function; and the Controls (C) enter the box from the top and constrain the function.

In particular, Inputs and Outputs of the herein-depicted framework pertain to data and decisions, whereas Mechanisms correspond to specific algorithms, models, and means that allow creation of the RAM database, to model the reliability of WTs and to select the optimal maintenance strategy. The function application is constrained by the Controls, which in this research represents business aspects, data requisites, or maintenance process specificities. By defining the INCOMs in such manner, the technical framework flow can be conceived as a flow in which the output of a function constitutes the input of another until an optimal maintenance strategy is reached. In the successive subsections, each one of the functions with their corresponding INCOMs is thoroughly explained, besides Table 1 gathers all the mathematical symbols utilized in the subsections. 
Table 1. Nomenclature.

\begin{tabular}{|c|c|c|c|}
\hline \multicolumn{4}{|c|}{ Reliability Modeling } \\
\hline$\beta$ & $\begin{array}{l}\text { Shape parameter of Weibull } \\
\text { distribution }\end{array}$ & $d_{i}$ & Diagonal entries of Degree matrix \\
\hline$\alpha$ & $\begin{array}{l}\text { Scale parameter of Weibull } \\
\text { distribution }\end{array}$ & $\overline{\bar{I}}$ & Identity matrix \\
\hline$\gamma$ & Euler-Mascheroni constant & $L_{\text {sym }}$ & Graph Laplacian matrix \\
\hline$\Gamma$ & Gamma function & $\lambda_{1}, \ldots, \lambda_{C}$ & Graph Laplacian matrix eigenvalues \\
\hline$\overline{\bar{W}}$ & Similarity matrix & $\bar{u}_{1}, \ldots, \bar{u}_{C}$ & Graph Laplacian matrix eigenvectors \\
\hline$w_{i j}$ & Entries of the similarity matrix & $\overline{\bar{U}}$ & Matrix of eigenvectors \\
\hline$d_{K L}\left(\mu_{i} \| \mu_{j}\right)$ & $\begin{array}{l}\text { Kullback-Leibler Divergence among } \\
\text { two distributions }\end{array}$ & $u_{i c}$ & Entries of $\overline{\bar{U}}$ matrix \\
\hline$d_{K L}^{s y m}$ & $\begin{array}{l}\text { Symmetric Kullback-Leibler } \\
\text { Divergence }\end{array}$ & $\overline{\bar{T}}$ & Transformed $\overline{\bar{U}}$ matrix \\
\hline$\overline{\bar{D}}$ & Degree matrix & $t_{i c}$ & Entries of $\overline{\bar{T}}$ matrix \\
\hline \multicolumn{4}{|c|}{ Maintenance Strategy Definition } \\
\hline$k_{a}$ & Time value for money constant & $G P_{t}$ & Generated power in $t$ \\
\hline$q^{c}$ & Restoration factor of CM & NT & Number of MTs \\
\hline$q^{p r}$ & Restoration factor of PM & $c^{\text {disp }}$ & Cost of dispatching a team \\
\hline$c^{c}$ & Corrective cost & $\theta_{t}$ & $\begin{array}{l}\text { Binary variable for corrective dispatch } \\
\text { of MT }\end{array}$ \\
\hline$c^{p r}$ & Preventive cost & $\gamma_{t}$ & $\begin{array}{l}\text { Binary variable for preventive } \\
\text { dispatch of MT }\end{array}$ \\
\hline$c^{n a}$ & $\begin{array}{l}\text { Opportunity cost for not-produced } \\
\text { energy }\end{array}$ & $c^{\text {team }}$ & Cost of MT \\
\hline$c^{p}$ & Penalty cost & $D R T$ & Dynamic reliability threshold \\
\hline$z_{\text {hikt }}$ & Binary variable for $\mathrm{CM}$ & $S R T$ & Static reliability threshold \\
\hline$y_{\text {hikit }}$ & Binary variable for PM & $T^{w t}$ & Available working time of MTs \\
\hline$m^{c}$ & Maintainability of corrective actions & $t$ & Time period (days) \\
\hline$m^{p r}$ & Maintainability of preventive actions & & \\
\hline
\end{tabular}

\subsection{RAM Database Creation}

Information is an essential pillar in every decision-making process and it is highly conditioned by the characteristics of the data it comes from. In the decision-making process, to select the optimal maintenance strategy herein presented, the information utility will be highly conditioned by the quantity and quality of the available data (Constraints $C 1$ and C2). As stated by [21], many data are recorded for maintenance management purposes rather than reliability; hence, the information content may be misleading without the proper scrutiny and cleaning. In the proposed technical framework, building up the RAM database from the data coming from the SCADA (Supervisory Control And Data Acquisition) and the CMMS (Computerized Maintenance Management Software) systems is considered.

- $\quad$ SCADA Data (Input I1): this data is intended to provide feedback of a high-level overview of the performance of the WTs. The data coming from the SCADA system can be categorized into three types of information recorded in time intervals: operational data, availability data, and alarms data. The operational data usually pertains to different variables which characterize the operation of the WTs, e.g., power output, wind speed, temperature of components, or environmental conditions. Availability is a measure of the total time the WT is operational and ready to produce power, independently of external factors such as the weather, the grid state, or maintenance activities. In addition, the data coming from the alarms is sensor information which indicates the state of the WTs and have an associated severity level and usually are responsible for triggering corrective maintenance actions. 
- CMMS Data (Input I2): generally the operators of WFs keep track of the maintenance actions performed in the WTs in a variety of forms. This record of maintenance activities is usually known as Work Orders or Maintenance Logs. The work orders may be handwritten in predefined forms, but presently it is more common to find the data as digital work order inputs which have detailed information regarding the materials and resources consumed.

To construct a proper RAM database from this data, it is essential to invest a considerable amount of effort in filtering the data, bearing in mind the objective of the data. Therefore, a proper determination of failure modes underpins the data-quality enhancement (Mechanism M1). As the data comes from two different sources, it is essential to match the information contained in both. With that aim, the identification of the patterns that link SCADA information with the maintenance work orders of the CMMS (Mechanism M2) is proposed. Once the data is properly filtered according to the failure mode definition and the coherence among the two sources of data is ensure, it is possible to calculate the Time Between Failures (TBF) for the failure modes of the WTs (Mechanism M3).

\subsection{Reliability Modeling}

Considering that the database contains the failure information (output from previous functional box O1) with certain quality (Constraint C3), it is possible to address the modeling of the reliability following a spectral cluster approach, which is proposed to address the differences in the failure behaviors of the same failure mode in each WT (Constraints C4 and C5). The methodological process followed in this functional unit is represented in Figure 2.

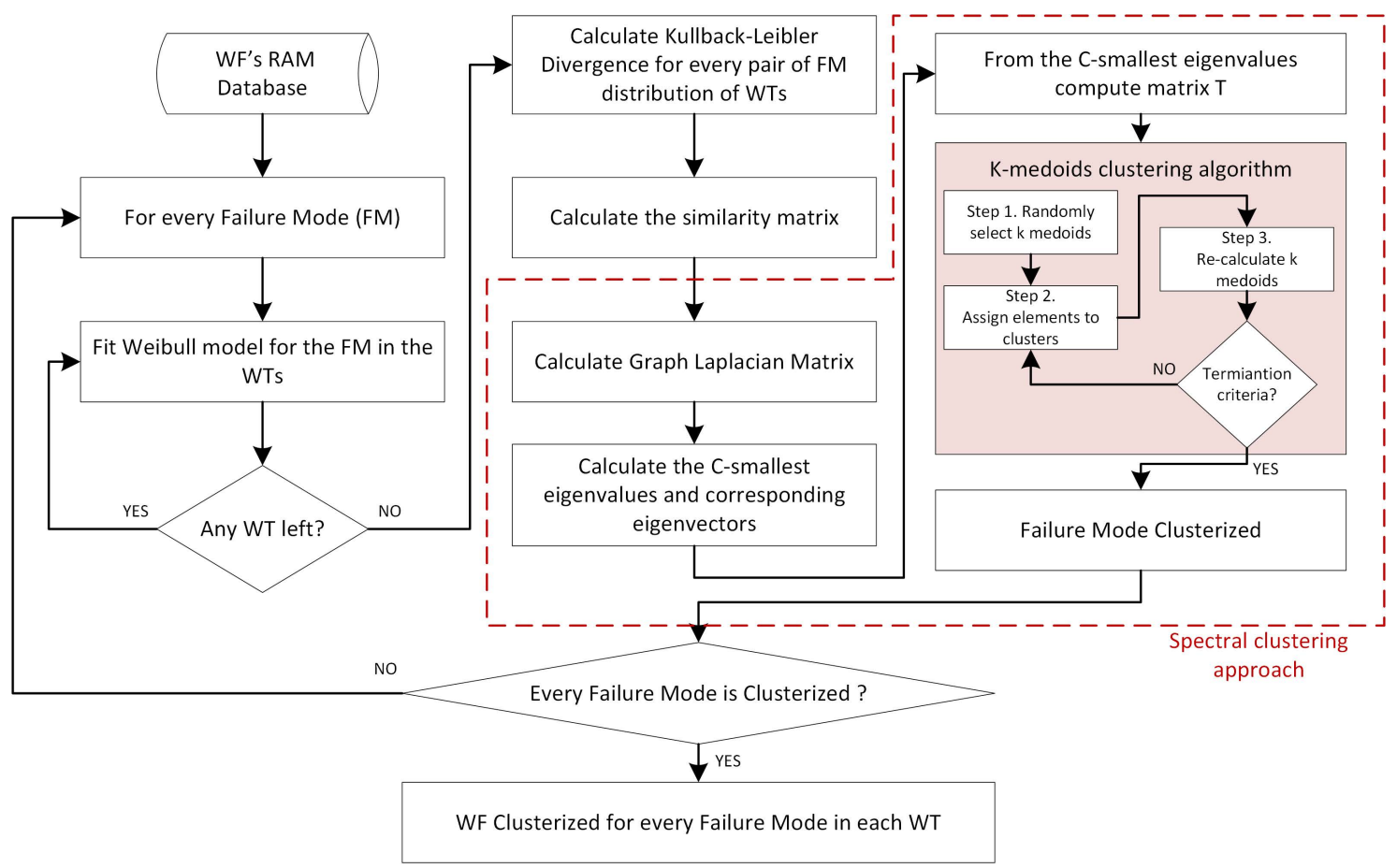

Figure 2. Methodological process of the Reliability Modeling functional box.

For every failure mode, the process considers fitting a Weibull distribution to the TBF data for each WT (Mechanism M4). With the Weibull distributions of each WT and failure mode, it is the purpose of the algorithms herein presented to cluster them following a similarity-based approach inspired by the work in $[26,27]$.

It is possible to measure how similar two probability distributions are according to the Kullback-Leibler Divergence (Mechanism M5), which can be calculated by Equation (1) in the case of two Weibull distributions being $\gamma \approx 0.5772$ the Euler-Mascheroni constant and $\Gamma=\int_{-\infty}^{+\infty} t^{z-1} e^{-t} d t z \geq 0$ the 
gamma function. Nonetheless the Kullback-Leibler Divergence is a non-symmetric measure which can be converted to symmetric by Equation (2) and therefore the similarity among two Weibull distributions can be described in a generic and symmetric form by Equation (3). Expressing similarities in such way allows the definition of a similarity matrix $\overline{\bar{W}}$ in which each element is $w_{i j}$.

$$
\begin{gathered}
d_{K L}\left(\mu_{i} \| \mu_{j}\right)=\log \left(\frac{\beta_{i} \alpha_{j}^{\beta_{j}}}{\beta_{j} \alpha_{i}^{\beta_{i}}}\right)-\left(\beta_{i}-\beta_{j}\right)\left(\log \left(\alpha_{i}\right)-\frac{\gamma}{\beta_{i}}\right)+\left(\frac{\alpha_{i}}{\alpha_{j}}\right)^{\beta_{j}} \Gamma\left(\frac{\beta_{j}}{\beta_{i}}+1\right)-1 \\
d_{K L}^{\text {sym }}=\frac{1}{2}\left(d_{K L}\left(\mu_{i} \| \mu_{j}\right)+d_{K L}\left(\mu_{j} \| \mu_{i}\right)\right) \\
w_{i j}=\frac{1}{1+d_{K L}^{\text {sym }}}
\end{gathered}
$$

From the similarity matrix $\overline{\bar{W}}$ of size $[N, N]$ it is possible to construct the graph $G=(V, E)$ where each of the nodes $v_{i}$ represents the baseline Weibull distribution of certain failure mode of each turbine, and each edge $e_{i j}$ is the similarity between two distributions based on the Kullback-Leibler Divergence. Having defined such a graph, the problem is finding the partition of the graph such that the weights of the edges are small and large for intercluster and intracluster connections, respectively. To fulfil such an aim, the spectral clustering approach is proposed, therefore it is necessary to compute the normalized Graph Laplacian matrix. From $\overline{\bar{W}}$ the degree matrix $\overline{\bar{D}}$ is calculated, which is a diagonal matrix whose diagonal entries are defined according to Equation (4), and then the normalized Graph Laplacian matrix is calculated as described in Equation (5), where $\overline{\bar{L}}=\overline{\bar{D}}-\overline{\bar{W}}$ and $\overline{\bar{I}}$ is the identity matrix of size $[N, N]$.

$$
\begin{gathered}
d_{i}=\sum_{j=1}^{N} w_{i j}, \quad i=1, \ldots, N \\
L_{s y m}=\overline{\bar{D}}^{-1 / 2} \overline{\bar{L}}^{-1 / 2}=\overline{\bar{I}}-\overline{\bar{D}}^{-1 / 2} \overline{\bar{W}}^{-1 / 2}
\end{gathered}
$$

To extract the information of the graph, the $C$ smallest eigenvalues $\lambda_{1}, \ldots, \lambda_{C}$ are selected along with their corresponding eigenvectors $\bar{u}_{1}, \ldots, \bar{u}_{C}$, with $C$ the desired number of clusters. The relevant information is considered by transforming matrix $\overline{\bar{W}}$ into a reduced matrix $\overline{\bar{U}}$ of size $[N, C]$. The columns of $\overline{\bar{U}}$ are the $C$ eigenvectors $\bar{u}_{1}, \ldots, \bar{u}_{C}$ which contain the information regarding the similarities among the i-th baseline distribution and others. It has been proven that it is possible to enhance cluster properties of the data by normalizing the rows of the matrix $\overline{\bar{U}}$ and forming matrix $\overline{\bar{T}}$ [52], where every element is computed following Equation (6).

$$
t_{i c}=\frac{u_{i c}}{\left(\sum_{c=1}^{c} u_{i c}^{2}\right)^{0.5}}, i=1, \ldots, N, c=1, \ldots, C
$$

Once the matrix $\overline{\bar{T}}$ is obtained, a k-medoids algorithm (Mechanism M6) is proposed as an unsupervised clustering to partition the dataset into $C$ clusters, which has proven to perform better for large datasets and to be more stable against possible outliers [53].

By repeating these calculations for every failure mode, it is possible to address the definition of the maintenance strategy of a WF. The effectiveness of the maintenance strategy is expected to increase since the WF is now characterized by different clusters (Output O4) of every failure mode, with their corresponding reliability model (Output O5) which directly tackles the issue of heterogeneity in the failure frequencies. 


\subsection{Maintenance Strategy Definition}

The clustering and their corresponding reliability models will support the definition and optimization of the maintenance strategy. In line with the insights provided in the literature review, opportunistic maintenance management is especially suitable for the wind energy sector, since it takes advantage of short-term information and dependencies among the WTs to make optimal maintenance decisions. Accordingly, the framework proposes to implement a reliability-based dynamic opportunistic maintenance policy (C8) summarized in Figure 3, which as well as considering the economic dependencies of WTs, enables taking advantage of more favorable weather conditions to enhance WF production outcome. To fulfil this aim, based on the concept of dynamic reliability thresholds-acting as decision variables of the maintenance model — maintenance activities are fostered at low wind-speed periods, and hindered at high wind-speed periods, thus limiting the production losses caused by maintenance downtimes.

While the interested reader may address the dynamic opportunistic maintenance policy in [5], the building blocks of the multi-level maintenance optimization model developed for this research are summarized as follows (the definition of the intermediate variables of the model is in Table 2):

- Problem definition. The wind farm (WF) to be considered in the case study involves the maintenance of $\mathrm{H}$ wind turbines (WTs) and their systems $(i=1,2, \ldots, N)$, connected in series. Each of the systems might fail in $\mathrm{k}$ different failure modes (FMs), which are classified according to their consequences and require different corrective maintenance $(\mathrm{CM})$ activities $(k=1,2, \ldots, K)$. Likewise, WF managers may decide to maintain the WTs' system before failure occurrence, where per-FM different preventive maintenance (PM) levels can be performed $(j=1,2, \ldots, J)$. This classification depends on the restoration factor $(q)$. If the PM activity restores the system to a state in which its operating and reliability behavior is as good as new, i.e., replacement activities, it is considered to be perfect maintenance $(j=J)$. On the contrary, when PM activity partially restores the condition of the system to an operational condition worse than the new one but better than just before the performance of maintenance, it is considered to be imperfect maintenance (see [54] for further information), with $j=1$ the most imperfect repair.

- Objective functions. The main objectives pursued by the maintenance strategy are defined. They should be aligned with the objectives of the business (C9) and should consider the whole lifecycle of the assets. In the case of WFs, special emphasis is placed on optimizing the OPEX (considering time value of money by means of $k_{a}$ ) and production losses entailed by the maintenance strategy (See Equations (12) and (13)). The core parts of these indicators are:

- Corrective and preventive cost (I3), subjected to the restoration effect $(q)$ of the maintenance activity carried out, as in [34,49]. They consider the materials and tools needed to perform them $\left(c_{i k}^{c}, c_{i k}^{p r}\right)$, as well as the opportunity cost that they entail, represented by the amount of power that could not be produced because of performing such maintenance activities $\left(c^{n a}\right)$. Likewise, in the case of $\mathrm{CM}$, failures usually avoid distribution of the committed energy, entailing a penalty $\operatorname{cost}\left(c^{p}\right)$.

$$
\begin{gathered}
z_{h i k t} \cdot\left[c_{i k}^{c} \cdot\left(q_{i k}^{c}\right)^{2}+m_{i k}^{c} \cdot G P_{t} \cdot\left(c^{n a}+c^{p}\right)\right] \\
y_{h i k j t} \cdot\left[c_{i k j}^{p r} \cdot\left(q_{i k j}^{p r}\right)^{2}+m_{i k j}^{p r} \cdot G P_{t} \cdot c^{n a}\right]
\end{gathered}
$$

- Maintenance resources cost (I3). They consider the number of maintenance teams hired (NT) and the cost entailed by dispatching them to the WFs $\left(c^{\text {disp }}\right)$ either preventively $\left(\gamma_{t}\right)$ or correctively $\left(\theta_{t}\right)$.

$$
\begin{gathered}
\left(\gamma_{t}+\theta_{t}\right) \cdot c^{\text {disp }} \\
N T \cdot c^{\text {team }}
\end{gathered}
$$


- Production losses. They consider the maintainability (O3) of PM and CM activities $\left(m_{i k}^{c}, m_{i k}^{p r}\right)$ as well as the power that would have been generated $\left(G P_{t}\right)$.

$$
G P_{t} \cdot\left(m_{i k}^{c} \cdot z_{h i k t}+m_{i k j}^{p r} \cdot y_{h i k j t}\right)
$$

- Maintenance strategy. An imperfect maintenance strategy where decisions are triggered by dynamic reliability thresholds is defined (see Equation (14)):

1. $D R T_{i k t}$, if the reliability of any of the FMs (O5) is below this threshold, a maintenance team is compulsorily dispatched to the WFs to perform PM.

2. $S R T_{i k j t}$, once a maintenance team is dispatched to the WF, either preventively or correctively, and according to the reliability of the specific FM (O5), it determines whether PM level $j$ should be performed during period $t$ for preventing FM $k$ of system $i$.

- Capacity constrains (C8). Maintenance teams' availability and their working time $\left(T^{w t}\right)$ is defined (see Equation (15)).

- Maintenance process constraints (C7). Only one maintenance task per WT and time period is allowed (see Equation (16)).

Once the building block of the model has been defined, the mathematical formulation of the model is expressed in the following equations regarding the objective functions $(O F)$, the constraints of the model, and the intermediate binary variables (in Table 2).

$$
\begin{gathered}
\text { OF } F_{\text {opex }}=\min \left[\sum_{t}\left(\gamma_{t}+\theta_{t}\right) \cdot c^{\text {disp }}+\sum_{h} \sum_{i} \sum_{k} \sum_{t} z_{h i k t}\left[c_{i k}^{c}\left(q_{i k}^{c}\right)^{2}+m_{i k}^{c} \cdot G P_{t}\left(c^{n a}+c^{p}\right)\right]+\right. \\
\left.\sum_{h} \sum_{i} \sum_{k} \sum_{j} \sum_{t} y_{h i k j t}\left[c_{i k j}^{p r}\left(q_{i k j}^{p r}\right)^{2}+m_{i k j}^{p r} \cdot G P_{t} \cdot c^{n a}\right]+\sum_{t} N T \cdot c^{\text {team }}\right] \cdot\left(1+k_{a}\right)^{-t} \\
O F_{L P}=\min \sum_{t} G P_{t} \cdot\left(\sum_{h} \sum_{i} \sum_{k} m_{i k}^{c} \cdot z_{h i k t}+\sum_{h} \sum_{i} \sum_{k} \sum_{j} m_{i k j}^{p r} \cdot y_{h i k j t}\right)
\end{gathered}
$$

S.T.

$$
\begin{gathered}
0 \leq D R T_{i k t} \leq S R T_{i k 1 t} \leq \ldots \leq S R T_{i k j t} \leq \\
\leq \ldots \leq S R T_{i k J t} \leq 1 i \epsilon I, k \in K, j \in J ; t \epsilon T \\
\sum_{i} \sum_{k} \sum_{j} m_{i k j}^{p r} \cdot y_{i k j t}+\sum_{i} \sum_{k} m_{i k}^{c} \cdot z_{i k t} \leq N T \cdot T^{w t} \forall t \in T \\
\sum_{j} y_{h i k j t}+z_{h i k t} \leq 1 h \epsilon H, i \in I, k \in K, t \epsilon T \\
z_{h i k t}, y_{h i k j t} \in\{0,1\} h \epsilon H, i \epsilon I, k \in K, t \in T, \forall j=1,2
\end{gathered}
$$

Table 2. Intermediate binary variables used in the model.

\begin{tabular}{ll}
$z_{\text {hikt }}= \begin{cases}1 \text { if CM } \text { is performed in systemi } \\
\text { of WThin period } t \\
0 & \text { otherwise }\end{cases}$ & $y_{\text {hikjt }}= \begin{cases}1 & \text { if PM jis performed in FM } \\
\text { kof systemi of WT hin period } t \\
0 & \text { otherwise }\end{cases}$ \\
$\theta_{t}= \begin{cases}1 & \text { if a MT is correctively dispatched to WF } \\
\text { in period } t & \text { otherwise }\end{cases}$ & $\gamma_{t}= \begin{cases}1 & \text { if a MT is preventively dispatched } \\
\text { to WF in period } t & \text { otherwise }\end{cases}$ \\
\hline
\end{tabular}


Due to the numerous stochastic processes that must be considered within maintenance management models, such as repair processes or climate conditions, it is difficult to solve them analytically. Therefore, in line with previous research $[46,49,55]$, the analytically derived model has been implemented in a simulation model (M7) that enables both the maintenance processes characterization and its optimization. The specific simulation and decision processes, as well as the restrictions considered, may be addressed by the reader in the flowchart of Figure 3.

As may be noticed, maintenance decisions are triggered according to the dynamic reliability thresholds (conditioned by weather conditions), which define the maintenance strategy (C6). Whenever a maintenance activity is performed, their reliability is updated, as are the values of the OF considered (C9). Likewise, specific maintenance processes (C7) and available resources (C8) are considered to analyze whether maintenance activities may be triggered. Such decision processes are repeated for each time period, until the end of the WT lifecycle, where the final OF are achieved.

Since more than one objective is pursued by the modeled maintenance problem, i.e., minimizing OPEXs and production losses, multi-objective optimization algorithms (M8) must be implemented to solve them. To this respect, the multi-objective meta-heuristic NSGA II [56] has been implemented, which offers high-quality non-dominated solutions and diversity on the Pareto Front [57].

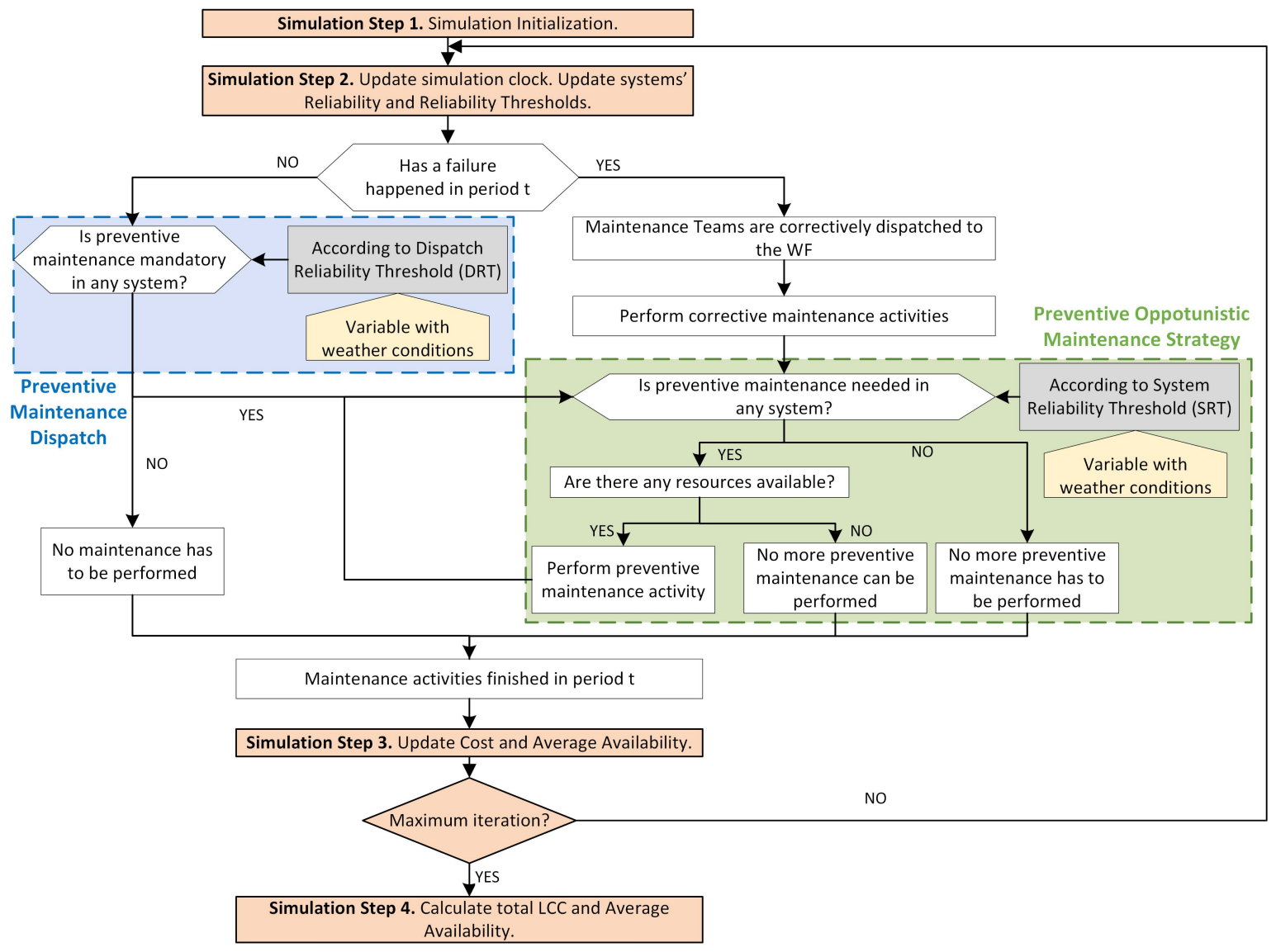

Figure 3. Dynamic Opportunistic Maintenance simulation flowchart.

In this context, the optimal maintenance strategies will be found by a joint use of the simulation model developed and the NSGA II optimization algorithm. While the former allows evaluation of the outcome of the selected maintenance strategy according to the OF, the latter will guide what maintenance strategies should be selected following the logic underlying behind the algorithm. Once the optimization process is finished, the aforementioned Pareto Front will be obtained with its corresponding Key Performance Indicators (KPI). From the Pareto Front and according to the 
lifecycle KPIs (M9), decision-makers will be able to represent their decision preferences through the maintenance strategy they choose (O6).

\section{Case Study}

To test the suitability of the proposed research presented in the paper, a case study based on real data is presented. The case study is especially focused on the reliability modeling and the maintenance strategy definition so the readers can see how to apply the proposed algorithms to manage the maintenance of the fleet. The proposed approach is compared in the case study against a static opportunistic maintenance policy which is in itself an advanced maintenance policy. By the application of the clustering approach and the dynamic opportunistic maintenance, it can be seen that the good results rendered by the static opportunistic maintenance can be further improved.

\subsection{Description}

The application of the technical framework is considered within a case study of an onshore WF consisting of 100 WTs $(H=100)$ whose behavior has been simulated for 20 years based on real field data. The real data correspond to over 300 WTs of 1.67 megawatt (MW) operating in the north of Spain for a time span of 12 years. According to the importance of wind speed for the energy-based availability and the dynamic opportunistic maintenance model, the simulation has been fed with wind data from the location assumed.

RAM data has been provided by a wind energy OEM and pertains to eight FMs. The FMs correspond to minor and major failures $(K=2)$ of four components $(N=4)$ which are critical from the maintenance perspective, either in terms of availability or cost: the gearbox, the blades, the yaw system, and the pitch system. Likewise, for each failure mode, both perfect and imperfect maintenance levels are considered $(J=2)$, where perfect maintenance has a restoration factor of $q_{i k 2}^{p r}=1$ and imperfect maintenance of $q_{i k 1}^{p r}=0.75$, according to the maintenance routine adopted.

In terms of the cost structure required to analyze the maintenance management from a lifecycle perspective, the main costs considered are as follows. The maintenance team costs, consisting of 2 workers each, are assumed to be $800 € /$ day, the opportunity cost $105 € / \mathrm{MWh}$, and the penalization cost $35 € /$ MWh. Likewise, the reader may address the specific material costs considered in [58]. Cost of PM is assumed to be lower than CM to avoid obvious results ( $30 \%$ lower). Finally, an interest rate of $5 \%$ has been determined for the LCC analysis.

\subsection{Results}

From the simulated database, the behavior of each one of the FMs of every WT has been characterized by fitting a two-parameter Weibull distribution. Following the proposed methodology, the differences among each of the WTs for every failure mode have been assessed by means of the Kullback-Leibler Divergence. This enables the expression of the similarities among the turbines for each failure mode by the weight matrix, which can be represented as an undirected graph. Then, for each failure mode graph, the partition that maximizes the similarities among elements in the same cluster and minimizes the similarities outside the cluster has been found by the k-medoids algorithm. The partition provides the number of clusters for every failure mode, hence each cluster is formed of WTs with like behaviors for each failure mode. For every cluster, a joint reliability Weibull model can be fitted for the failure data that correspond to the WTs in the cluster; the model with which they will be managed will be developed later.

The partitions of the FMs have also been found by the k-means algorithm to compare the performance of both clustering algorithms. The results yielded by the k-means algorithm are very similar to the results yielded by the k-medoids. In fact, 11 out of 31 clusters remain the same. In the differing clusters, the practical deviations are very slight; the usage of one algorithm over the other entails differences of $0.49 \%$ and $2.77 \%$ on average in the obtained scale and shape parameters, respectively. Therefore, the remainder of the case study, consisting of the simulation-based optimization, has been carried out with the results 
yielded by the k-medoids algorithm, since it is more stable against outliers and performs better for large datasets according to previously cited literature.

In Figure 4, as an example, the undirected graph of the minor gearbox failure of every WT is represented. In it can also be seen the reliability functions corresponding to every cluster. In the depicted example, six clusters can be appreciated and the nodes in each cluster correspond to the WTs identified by a number. It can be seen that the edges connecting the nodes have different color intensity, which corresponds to the weight that quantifies the similarity among the behaviors of the same failure mode in different turbines, i.e., the higher the intensity, the more alike they are. By this representation, it can also be seen from a general perspective the similarities among the clusters. The reliability functions corresponding to each one of the clusters can also be seen in Figure 4, and it can be seen how the closer two are, the higher the intensity of the connections of the nodes from the clusters in the graph.
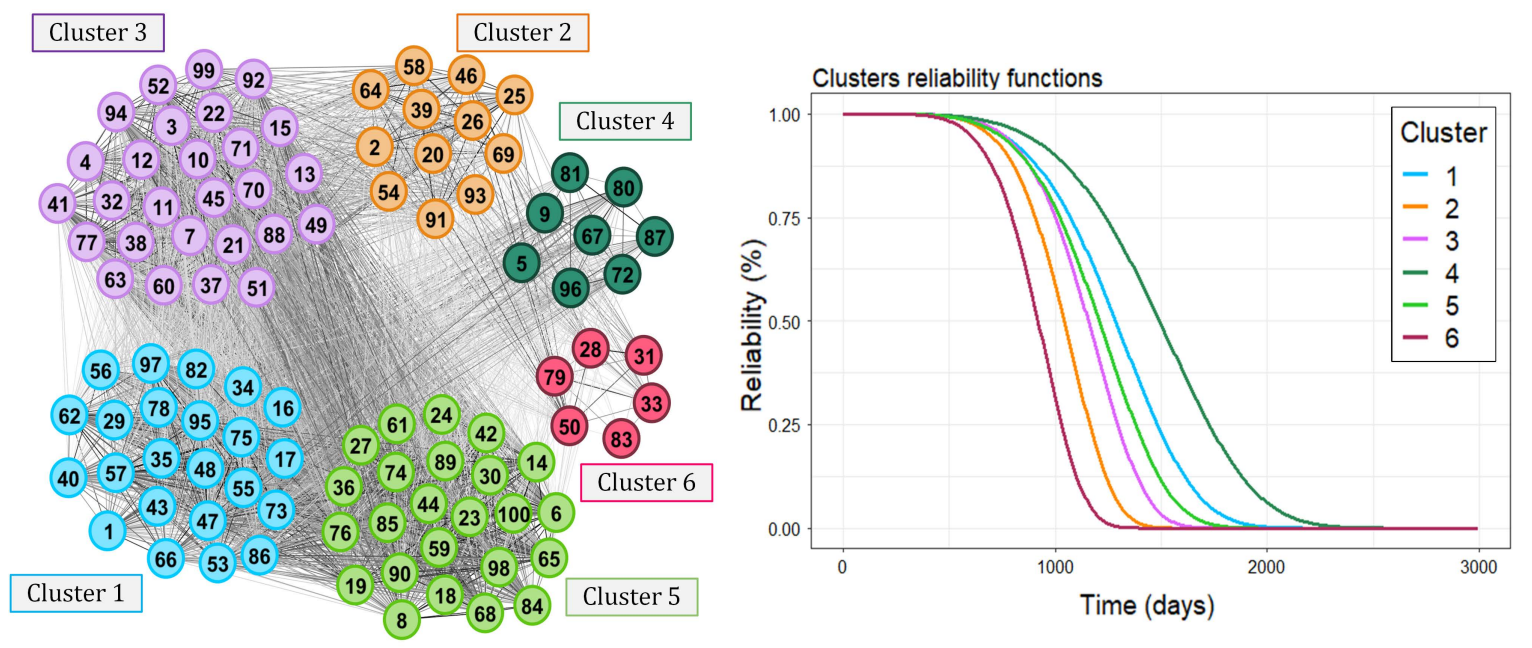

Figure 4. Clusterization example. Minor failure mode of the gearbox.

The results obtained for the clusterization of each one of the FMs have been summarized in Table 3. In the table, the components and the FMs of the number of clusters can be seen, as well as the number of WTs belonging to every cluster. The definition of minor and major FMs has been made according to the repairing details:

- Minor repairing. It is provoked by a system failure that implies a maintenance team is dispatched for repairing. Costs of materials and tools are not very high, as minor components are going to be repaired. Thus, there is a reparation impact on system lifecycle but it is neither too high in terms of time, nor too costly. Repair time varies between 4 and $24 \mathrm{~h}$.

- Major repairing. Replacement of important components or complete systems is done. Therefore, repairs have a great impact on system lifetime, being able to return the system state to an as-good-as-new state. Costs of materials and tools are high, and repair time is above $24 \mathrm{~h}$.

From Table 3, and also the graph of Figure 4, it is possible to conclude that the proposed approach is stable when identifying clusters with a low number of nodes in it. This is an important implication, since it is possible to identify small numbers of turbines behaving differently, and sometimes this may imply a cause which can be addressed or benchmarked.

Once the behaviors of the FMs in the WF have been assessed, it is possible to define the maintenance strategy according to the reliability of each one of the clusters of every failure mode. In the framework, the proposed maintenance strategy is an opportunistic maintenance policy defined by a simulation-based optimization. The optimization is multi-objective so the two-fold aforementioned problem of reducing maintenance costs is addressed. The simulation-based optimization provides several non-dominated solutions, which entails a trade-off among costs and production loss. With the 
solutions rendered by the optimization, it is possible to construct a Pareto Front in which the maintenance strategy can be selected according to the trade-off desired by the customers.

Table 3. Clusterization results.

\begin{tabular}{cccc}
\hline Component & Failure Mode & Number of Clusters & Number of WTs in Each Cluster \\
\hline \multirow{2}{*}{ Gearbox } & Minor & 6 & $23-12-27-8-24-6$ \\
& Major & 3 & $52-27-21$ \\
\hline \multirow{2}{*}{ Blades } & Minor & 4 & $45-33-18-4$ \\
& Major & 2 & $39-61$ \\
\hline \multirow{2}{*}{ Yaw } & Minor & 4 & $34-33-17-16$ \\
& Major & 3 & $30-47-23$ \\
\hline \multirow{2}{*}{ Pitch } & Minor & 5 & $24-27-28-7-14$ \\
& Major & 3 & $29-20-51$ \\
\hline
\end{tabular}

In Figure 5, a comparison of two Pareto optimals is presented. One is obtained by addressing the failures through the clusterization and then defining maintenance strategy through dynamic opportunistic policy; the other one is obtained through a static opportunistic policy with a generic reliability Weibull model for each failure mode of the turbines. In Figure 5a, it can be seen that the proposed approach provides solutions which are unreachable for the static opportunistic policy. Furthermore, all the solutions provided by the clusterization with the dynamic opportunistic maintenance strategy are better in terms of costs and production loss. As an example to provide further insights, arbitrary customer requisites have been defined, which correspond to 122,000,000€ as a maximum OPEX and 120,000 MW/h lost for the 20 years of the lifecycle of the WF. In Figure $5 b$ it can be seen that these requisites defined a feasible area of options and their intersection sets where is the Minimum Viable Offer (MVO).

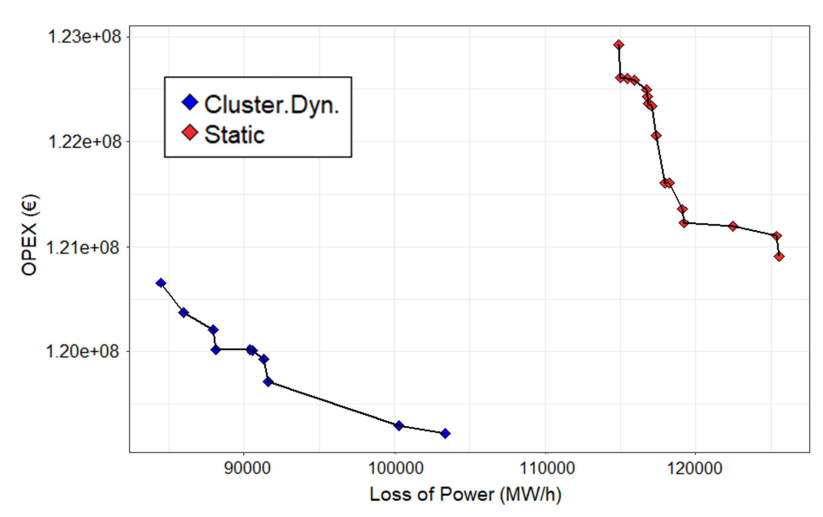

(a) Comparison of Pareto optimals

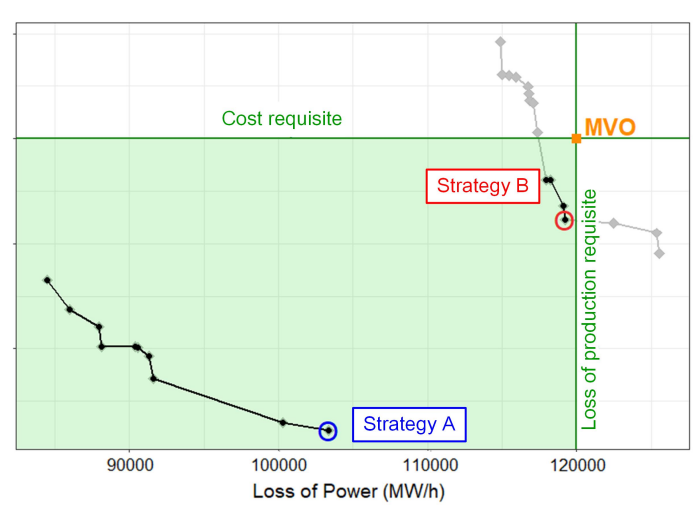

(b) Feasible strategies for customer's requisties

Figure 5. Pareto Front and Pareto Front with customer requisites.

According to the strategies represented in Figure 5, two of them have been selected (one from each policy) to compare their lifecycle performance. The selected strategies are the ones that fulfil the production loss requisite at a lower cost, and their performance in terms of OPEX and production loss is compared in Figure 6. It can be seen that the cost savings among the two strategies are over 3 million $€$, despite not being very significant. These savings come from the opportunity cost of producing energy at profitable wind-speed periods and from more accurate reliability estimates, which avoid some corrective actions. This result is reasonable and was expected since the dynamic opportunistic policy compared with the static does not reduce the amount of maintenance needed, but it performs it in more suitable moments. 


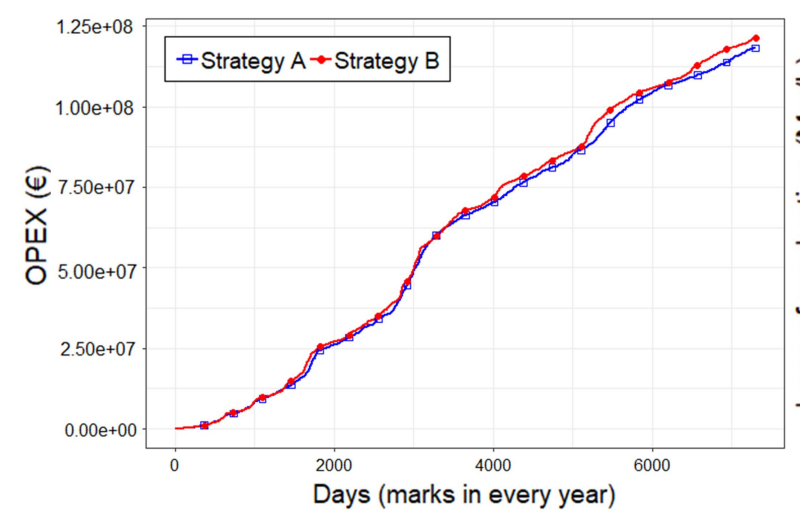

(a) OPEX comparison

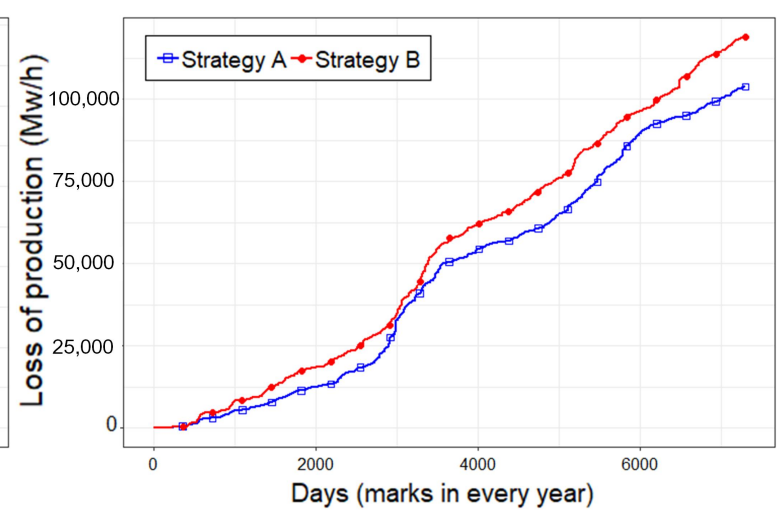

(b) Productions loss comparison

Figure 6. Performance comparison of costs (a) and production loss (b) of strategies A and B.

Accordingly, the production loss difference is more significant, reaching an improvement of $14.5 \%$ by the end of the lifecycle. This improvement in the production loss is due to the performance of maintenance activities in the most favorable wind conditions. Besides, the better reliability estimates enhance the calculation of the failure probability, enabling the avoidance of unexpected $\mathrm{CM}$, which takes more time to perform. Therefore, it can be seen that the proposed approach improves the performance of the static opportunistic maintenance with a general reliability model, not only in terms of cost, but in terms of production loss as well.

\section{Concluding Remarks}

In the present paper, a technical framework for managing the maintenance of WFs is proposed, which integrates three modules that depart from data considerations, providing reliability analysis tools to define the maintenance strategy. The framework considers the creation of a RAM database to provide a solid basis of information which is transformed into knowledge by reliability modeling. The second module aims at modeling the reliability of the WF; to do so, a clustering approach is proposed. By means of the clustering approach, the different behaviors of the WTs, which are caused by either different technical solutions or different working conditions, are properly addressed. This accurate description of the WF failures enables the definition of a more effective maintenance strategy, which is defined by a dynamic opportunistic policy optimization finding a trade-off among costs and production loss.

The suitability of the comprehensive framework has been validated through a case study based on real field data. The proposed approach has been tested against a static opportunistic maintenance which estimates the reliability of the WTs with a generic reliability model. It has been shown that by answering the research question, the integration of state-of-the-art techniques in the proposed framework provides a step forward in the achievement of maintenance excellence. The proposed approach outperforms the alternative in terms of cost and production loss. The improvements are due to two reasons: (i) the reliability estimates are more accurate because the behavior of the WTs is better characterized; and (ii) the maintenance activities are performed at the most convenient moments, fostering the maximization of energy production.

The research here presented is a step forward in the maintenance management of WFs due to its practical nature. However, to enhance its applicability and implementation in the wind energy industry, further efforts should focus on the integration of the strategy here presented with condition-based maintenance. Moreover, it would be interesting to also explore models that directly address the influence of changing operational conditions on the reliability of WTs. This holistic perspective entails potential benefits in the field of O\&M of WFs, and it is worth researchers' and industry practitioners' attention. 
Author Contributions: Conceptualization, J.I., A.C.M. and J.U.; Formal analysis, J.I. and A.E.; Investigation, J.I. and A.E.; Methodology, J.I.; Project administration, J.I.; Software, J.I.; Supervision, A.C.M. and J.U.; Validation, A.C.M. and J.U.; Writing—original draft, J.I.; Writing—review \& editing, A.C.M., J.U. and A.E.

Funding: This research work was performed within the context of SustainOwner EmaitekPlus 2018-2019 Program of the Basque Government.

Conflicts of Interest: The authors declare no conflict of interest.

\section{Abbreviations}

The following abbreviations are used in this manuscript:

$\begin{array}{ll}\text { WF } & \text { Wind Farm } \\ \text { LCoE } & \text { Levelized Cost of Energy } \\ \text { CAPEX } & \text { Capital Expenditure } \\ \text { OPEX } & \text { Operational Expenditure } \\ \text { O\&M } & \text { Operations \& Maintenance } \\ \text { WT } & \text { Wind Turbine } \\ \text { RAM } & \text { Reliability, Availability, and Maintainability } \\ \text { LCC } & \text { Life-cycle Cost } \\ \text { INCOM } & \text { Inputs, Mechanisms, Controls and Outputs } \\ \text { SCADA } & \text { Supervisory Control And Data Acquisition } \\ \text { CMMS } & \text { Computerized Maintenance Management System } \\ \text { TBF } & \text { Time Between Failures } \\ \text { FM } & \text { Failure Mode } \\ \text { CM } & \text { Corrective Maintenance } \\ \text { PM } & \text { Preventive Maintenance } \\ \text { MT } & \text { Maintenance Team } \\ \text { NSGA } & \text { Non-dominated Sorted Genetic Algorithm } \\ \text { KPI } & \text { Key Performance Indicator } \\ \text { MW } & \text { Megawatt } \\ \text { MVO } & \text { Minimum Viable Offer }\end{array}$

\section{References}

1. Ackermann, T. Wind Power in Power Systems; Wiley-Blackwell: Hoboken, NJ, USA 2005; [CrossRef]

2. Asgarpour, M.; Sørensen, J.D. Bayesian Based Diagnostic Model for Condition Based Maintenance of Offshore Wind Farms. Energies 2018, 11, 300. [CrossRef]

3. Merizalde, Y.; Hernández-Callejo, L.; Duque-Perez, O.; Alonso-Gómez, V. Maintenance Models Applied to Wind Turbines. A Comprehensive Overview. Energies 2019, 12, 225. [CrossRef]

4. Zhao, Y.; Li, D.; Dong, A.; Kang, D.; Lv, Q.; Shang, L. Fault Prediction and Diagnosis of Wind Turbine Generators Using SCADA Data. Energies 2017, 10, 1210. [CrossRef]

5. Erguido, A.; Márquez, A.C.; Castellano, E.; Fernández, J.G. A dynamic opportunistic maintenance model to maximize energy-based availability while reducing the life cycle cost of wind farms. Renew. Energy 2017, 114, 843-856. [CrossRef]

6. Kaldellis, J.; Kapsali, M. Shifting towards offshore wind energy—Recent activity and future development. Energy Policy 2013, 53, 136-148. [CrossRef]

7. Byon, E. Wind turbine operations and maintenance: a tractable approximation of dynamic decision making. IIE Trans. 2013, 45, 1188-1201. [CrossRef]

8. Ozturk, S.; Fthenakis, V.; Faulstich, S. Assessing the Factors Impacting on the Reliability of Wind Turbines via Survival Analysis-A Case Study. Energies 2018, 11, 3034. [CrossRef]

9. Nielsen, J.S.; Sørensen, J.D. Bayesian Estimation of Remaining Useful Life for Wind Turbine Blades. Energies 2017, 10, 664. [CrossRef]

10. Leahy, K.; Gallagher, C.; O'Donovan, P.; O'Sullivan, D.T.J. Issues with Data Quality for Wind Turbine Condition Monitoring and Reliability Analyses. Energies 2019, 12, 201. [CrossRef] 
11. IRENA, I.R.E.A. Renewable Power Generation Costs in 2012: An Overview; International Renewable Energy Agency (IRENA): Abu Dhabi, UAE, 2012.

12. PowerTech eV, V. Levelised Cost of Electricity (LCOE 2015); Verfugbar unter; VGB PowerTech e.V.: Essen, Germany, 2015.

13. Jardine, A.K.; Tsang, A.H. Maintenance, Replacement, and Reliability: Theory And Applications, 1st ed.; CRC Press: Boca Raton, FL, USA, 2005; doi:10.1201/9781420044614.

14. Bertling, L.; Wennerhag, P. Wind Turbine Operation and Maintenance; Technical Report; Elforsk: Stockholm, Sweden, 2012.

15. Shafiee, M. Maintenance logistics organization for offshore wind energy: Current progress and future perspectives. Renew. Energy 2015, 77, 182-193. [CrossRef]

16. Izquierdo, J.; Márquez, A.C.; Uribetxebarria, J. Dynamic artificial neural network-based reliability considering operational context of assets. Reliabil. Eng. Syst. Saf. 2019, 188, 483-493. [CrossRef]

17. Izquierdo, J.; Crespo, A.; Uribetxebarria, J.; Erguido, A. Assessing the impact of operational context variables on rolling stock reliability. A real case study. In Safety and Reliability-Safe Societies in a Changing World; CRC Press, Ed.; Taylor \& Francis Group: London, UK; Trondheim, Norway, 2017; Proceedings of ESREL 2018, pp. 571-578.

18. Rausand, M.; Höyland, A. System Reliability Theory: Models, Statistical Methods, and Applications; John Wiley \& Sons Hoboken, NJ, USA, 2004; Volume 396.

19. Hameed, Z.; Vatn, J.; Heggset, J. Challenges in the reliability and maintainability data collection for offshore wind turbines. Renew. Energy 2011, 36, 2154-2165. [CrossRef]

20. Nguyen, T.H.; Prinz, A.; Friisø, T.; Nossum, R.; Tyapin, I. A framework for data integration of offshore wind farms. Renew. Energy 2013, 60, 150-161. [CrossRef]

21. Louit, D.; Pascual, R.; Jardine, A. A practical procedure for the selection of time-to-failure models based on the assessment of trends in maintenance data. Reliabil. Eng. Syst. Saf. 2009, 94, 1618-1628. [CrossRef]

22. Stamatelatos, M.; Veseley, W.; Dugan, J.; Fragola, J.; Minarik, J.; Rialsback, J. Fault Tree Handbook with Aerospace Applications; NASA Office of Safety and Mission Assurance: Washington, DC, USA, 2002.

23. Li, H.; Yang, C.; Zhao, B.; Wang, H.; Chen, Z. Aggregated models and transient performances of a mixed wind farm with different wind turbine generator systems. Electric Power Syst. Res. 2012, 92, 1-10. [CrossRef]

24. Cao, L.; Qian, Z.; Zareipour, H.; Wood, D.; Mollasalehi, E.; Tian, S.; Pei, Y. Prediction of Remaining Useful Life of Wind Turbine Bearings under Non-Stationary Operating Conditions. Energies 2018, 11, 3318. [CrossRef]

25. Zimroz, R.; Bartelmus, W.; Barszcz, T.; Urbanek, J. Diagnostics of bearings in presence of strong operating conditions non-stationarity-A procedure of load-dependent features processing with application to wind turbine bearings. Mech. Syst. Signal Process. 2014, 46, 16-27. [CrossRef]

26. Cannarile, F.; Compare, M.; Di Maio, F.; Zio, E. Handling reliability big data: A similarity-based approach for clustering a large fleet of assets. In Safety and Reliability of Complex Engineered Systems-Proceedings of the 25th European Safety and Reliability Conference; ESREL: Zurich,Switzerland, 2015; Volume 2015, pp. 891-896.

27. Cannarile, F.; Compare, M.; Di Maio, F.; Zio, E. A clustering approach for mining reliability big data for asset management. Proc. Inst. Mech. Eng. Part O J. Risk Reliabil. 2018, 232, 140-150. [CrossRef]

28. Baraldi, P.; Di Maio, F.; Rigamonti, M.; Zio, E.; Redouane, S. Unsupervised clustering of vibration signals for identifying anomalous conditions in a nuclear turbine. J. Intell. Fuzzy Syst. 2015, 28, 1723-1731. [CrossRef]

29. Jiang, B.; Pei, J.; Tao, Y.; Lin, X. Clustering uncertain data based on probability distribution similarity. IEEE Trans. Knowl. Data Eng. 2013, 25, 751-763. [CrossRef]

30. Ruiz de la Hermosa, R. Wind farm monitoring using Mahalanobis distance and fuzzy clustering. Renew. Energy 2018, 123, 526-540. [CrossRef]

31. Elijorde, F.; Kim, S.; Lee, J. A Wind Turbine Fault Detection Approach Based on Cluster Analysis and Frequent Pattern Mining. KSII Trans. Internet Inf. Syst. 2014, 8, 664-677. [CrossRef]

32. Hameed, Z.; Wang, K. Development of optimal maintenance strategies for offshore wind turbine by using artificial neural network. Wind Eng. 2012, 36, 353-364. [CrossRef]

33. Wang, H.; Pham, H. Optimal Preparedness Maintenance of Multi-unit Systems with Imperfect Maintenance and Economic Dependence. In Springer Series in Reliability Engineering; Springer Science + Business Media, Berlin, Germany: 2006; pp. 135-150. [CrossRef]

34. Ding, F.; Tian, Z. Opportunistic maintenance optimization for wind turbine systems considering imperfect maintenance actions. Int. J. Reliabil. Q. Saf. Eng. 2011, 18, 463-481. [CrossRef] 
35. Nicolai, R.; Dekker, R. A review of multi-component maintenance models. In Proceedings of the European Safety and Reliability Conference, Stavanger, Norway, 25-27 June 2007; pp. 289-296.

36. Dekker, R.; Wildeman, R.E.; van der Duyn Schouten, F.A. A review of multi-component maintenance models with economic dependence. Math. Methods Oper. Res. 1997, 45, 411-435. [CrossRef]

37. Sasieni, M.W. A Markov Chain Process in Industrial Replacement. OR 1956, 7, 148. [CrossRef]

38. Nakagawa, T.; Murthy, D. Optimal replacement policies for a two-unit system with failure interactions. RAIRO-Oper. Res. 1993, 27, 427-438. [CrossRef]

39. De Almeida, A.T.; Ferreira, R.J.P.; Cavalcante, C.A.V. A review of the use of multicriteria and multi-objective models in maintenance and reliability. IMA J. Manag. Math. 2015, 26, 249-271. [CrossRef]

40. Cavalcante, C.A.; Lopes, R.S. Multi-criteria model to support the definition of opportunistic maintenance policy: A study in a cogeneration system. Energy 2015, 80, 32-40. [CrossRef]

41. Cavalcante, C.; Lopes, R. Opportunistic Maintenance Policy for a System with Hidden Failures: A Multicriteria Approach Applied to an Emergency Diesel Generator. Math. Probl. Eng. 2014, 2014, 157282. [CrossRef]

42. Iqbal, M.; Azam, M.; Naeem, M.; Khwaja, A.; Anpalagan, A. Optimization classification, algorithms and tools for renewable energy: A review. Renew. Sustain. Energy Rev. 2014, 39, 640-654. [CrossRef]

43. Besnard, F.; Patrikssont, M.; Strombergt, A.B.; Wojciechowskit, A.; Bertling, L. An optimization framework for opportunistic maintenance of offshore wind power system. In Proceedings of the 2009 IEEE Bucharest PowerTech, Bucharest, Romania, 28 June-2 July 2009; [CrossRef]

44. Tian, Z.; Jin, T.; Wu, B.; Ding, F. Condition based maintenance optimization for wind power generation systems under continuous monitoring. Renew. Energy 2011, 36, 1502-1509. [CrossRef]

45. Ding, F.; Tian, Z. Opportunistic maintenance for wind farms considering multi-level imperfect maintenance thresholds. Renew. Energy 2012, 45, 175-182. [CrossRef]

46. Sarker, B.R.; Faiz, T.I. Minimizing maintenance cost for offshore wind turbines following multi-level opportunistic preventive strategy. Renew. Energy 2016, 85, 104-113. [CrossRef]

47. Zhu, W.; Fouladirad, M.; Bérenguer, C. A multi-level maintenance policy for a multi-component and multifailure mode system with two independent failure modes. Reliab. Eng. Syst. Saf. 2016, 153, 50-63. [CrossRef]

48. Atashgar, K.; Abdollahzadeh, H. Reliability optimization of wind farms considering redundancy and opportunistic maintenance strategy. Energy Convers. Manag. 2016, 112, 445-458. [CrossRef]

49. Abdollahzadeh, H.; Atashgar, K.; Abbasi, M. Multi-objective opportunistic maintenance optimization of a wind farm considering limited number of maintenance groups. Renew. Energy 2016, 88, 247-261. [CrossRef]

50. IEEE, C.S. IEEE Standard for Functional Modeling Language-Syntax and Semantics for IDEF0; IEEE Std 1320.1-1998; IEEE Computer Society: Washington DC, USA, 1998; p. i, [CrossRef]

51. Kusiak, A.; Larson, T.N.; Wang, J.R. Reengineering of design and manufacturing processes. Comput. Ind. Eng. 1994, 26, 521-536. [CrossRef]

52. Von Luxburg, U. A tutorial on spectral clustering. Stat. Comput. 2007, 17, 395-416. [CrossRef]

53. Velmurugan, T.; Santhanam, T. Computational complexity between K-means and K-medoids clustering algorithms for normal and uniform distributions of data points. J. Comput. Sci. 2010, 6, 363. [CrossRef]

54. Pham, H.; Wang, H. Imperfect maintenance. Eur. J. Oper. Res. 1996, 94, 425-438. [CrossRef]

55. Zille, V.; Bérenguer, C.; Grall, A.; Despujols, A. Modelling multicomponent systems to quantify reliability centred maintenance strategies. Proc. Inst. Mech. Eng. Part O J. Risk Reliabil. 2011, 225, 141-160. [CrossRef]

56. Deb, K.; Pratap, A.; Agarwal, S.; Meyarivan, T. A fast and elitist multiobjective genetic algorithm: NSGA-II. IEEE Trans. Evol. Comput. 2002, 6, 182-197. [CrossRef]

57. Salazar, D.; Rocco, C.M.; Galván, B.J. Optimization of constrained multiple-objective reliability problems using evolutionary algorithms. Reliabil. Eng. Syst. Saf. 2006, 91, 1057-1070. [CrossRef]

58. Martin-Tretton, M.; Reha, M.; Drunsic, M.; Keim, M. Data collection for current us wind energy projects: Component costs, financing, operations, and maintenance. Contract 2012, 303, 275-300. [CrossRef]

(C) 2019 by the authors. Licensee MDPI, Basel, Switzerland. This article is an open access article distributed under the terms and conditions of the Creative Commons Attribution (CC BY) license (http:/ / creativecommons.org/licenses/by/4.0/). 